\title{
Modeling of optical aberration correction using a liquid crystal device
}

\author{
Xinghua Wang ${ }^{\mathrm{a}}$, Bin Wang ${ }^{\mathrm{a}}$, ,Paul F. McManamon ${ }^{\mathrm{b}}, \mathrm{John}$ J. Pouch ${ }^{\mathrm{c}}$, Felix A. Miranda ${ }^{\mathrm{c}}$, \\ Philip J. Bos ${ }^{\mathrm{a}^{*}}$ \\ ${ }^{a}$ Liquid Crystal Institute, Kent State University, Kent, OH 44242 \\ ${ }^{\mathrm{b}}$ Air Force Research Lab, Dayton OH 45424-4653 \\ 'NASA Glenn Research Center, Cleveland, OH 44135
}

\begin{abstract}
Gruneisen ${ }^{1-3}$, has shown that small, light weight, liquid crystal based devices can correct for the optical distortion caused by an imperfect primary mirror in a telescope and has discussed the efficiency of this correction. In this paper we expand on that work and propose a semi- analytical approach for quantifying the efficiency of a liquid crystal based wavefront corrector for this application.
\end{abstract}

\section{INTRODUCTION}

Researchers at the Air Force Research Laboratory and other groups have pioneered in developing liquid crystal devices for tip-tilt, and more general wavefront correction ${ }^{[1-11]}$. McManamon and Watson et al. pioneered in liquid crystal Optical Phased Array device, as well as the basic efficiency theory related to such device ${ }^{[4]}$. Gruneisen et al, pioneered in high-resolution wavefront control in a large aperture telescope

\footnotetext{
*pbos@kent.edu, Tel: (330) 672-2511
} 
with diffractive optical element. They studied the diffraction efficiency, dynamic range of correction, efficiency for correcting large defocus and astigmatism, wavelength dependence of such system using Fourier Optics approach ${ }^{[1-3]}$. Also, Gruneisen has addressed the efficiency issue associated with the local wavefront slope needed for large correction.

Here, we present a simple model of evaluating the performance of a liquid crystal based wavefront corrector for the telescope correction application. Independent efficiency factors are introduced and discussed.

\section{A SIMPLE ANALYTICAL APPROACH FOR ESTIMATING THE CORRECTION EFFICIENCY}

We consider four major factors that contribute to the strehl ratio of the system after correction, as shown in Equation 1.

$$
\eta_{\text {total }}=\eta_{s} \eta_{\text {ray }} \eta_{\text {aber }} \eta_{S L M}
$$

The first factor, $\eta_{s}$, is associated with the passive loss in the system such as the imperfection in the mirror surface, scattering, absorption and front surface reflection loss etc. This factor mainly depends on the material and process that is used to make the optical elements.

The second efficiency factor , $\eta_{\text {ray }}$, is related to the non-conjugate position of the element being corrected relative to the corrector. In an ideal perfect conjugate system, where the correction device is 
placed at the conjugate position of the primary optical element, the position of any ray that hits a particular point on the primary mirror will hit a corresponding point on the LC-SLM. There is a one to one relationship of the ray interception position on the primary mirror and the correction device, and it doesn't matter what is the magnitude or order of the aberration on the primary mirror. In this case, $\eta_{\text {ray }}=1$ always holds true. However, this ideal conjugate relationship may not always hold true for a high numerical aperture telescope with large magnification. In this case, the wavefront aberration could cause substantial shift of the ray interception position on the correction device for different aberrations, and the efficiency $\eta_{\text {ray }}$ will be low. While other terms that govern the overall efficiency increase as the resolution of the correction device increases, this term drops because the significance of the non-conjugate nature of the system is greater if the considered resolution is higher. In can be seen that a ray will be more likely to shift to a wrong pixel on the correction device, if the pixel size of the correction device is small; which means, this efficiency term drops as the resolution of the correction device increases.

Not having an approach to quantify the efficiency term $\eta_{\text {ray }}$ with in a completely analytical method, we can use a simple ray tracing algorithm to describe this term in a physically meaningful way. Consider a certain aberration in a certain optical system, where we can trace a $\mathrm{m} \times \mathrm{m}$ bundle of rays throught it. The correction device in the system has $\mathrm{m} \times \mathrm{m}$ pixels across the clear aperture. For each pixel on the correction device, we can count how may rays intercepts the correction device on that pixel. When there is no aberration present in the system, the number of rays intercept a pixel on the correction device should be one. However, when aberration is present in the system, the number of rays intercepting a certain pixel is no longer one. We define $\mathrm{n}_{0}$ to be the number of pixels on the correction device that have no ray 
intercepting that pixel; $\mathrm{n}_{1}$, to the the number of pixels on the correction device that have one ray intercepting that pixel; $\mathrm{n}_{2}$, to be the number of pixels on the correction device that have two rays intercepting that pixel; and $\mathrm{n}_{\mathrm{k}}$, to be the number of pixels that have k rays intercepting that pixel. Since the efficiency of the system will be a direct function of how many rays will miss the desired pixel, which is simply $\mu_{0}$, it can be expressed in Equation (2).

$$
\eta_{\text {ray }}=1-\mu_{0}
$$

The third efficiency factor $\eta_{a b e r}$ is related to the resolution of the corrector. This term becomes larger as the resolution of the device becomes higher. If we assume an ideal piston-phase-plate correction device with finite resolution, the correction efficiency for random high order aberration is not $100 \%$. Consider the simplest case of correcting a simple tilt, and assume $\mathrm{q}$ is the number of steps for every wave of tilt, the efficiency factor $\eta_{a b e r}$ is the same as the diffraction efficiency for a stair-like blazed grating as in Equation $3^{[4]}$.

$$
\eta_{\text {aberr }}=\left(\frac{\sin (\pi / q)}{\pi / q}\right)^{2}
$$

We would like to estimate the efficiency factor that, like Equation 3, is related to the resolution of the correction device, but is applicable to the more general case of an arbitrary aberration.. Any aberration on the primary mirror will induce a corresponding but different aberration on the correction device. The aberration profile on the correcting device can be obtained by diffractive beam propagation of a Guassian beam from the primary optics to the correction device.. The whole aperture is divided into $1 \times 1$ small regions. Within region $\Omega$, if the region is very small, the aberration $P(x, y)_{x, y \in \Omega}$ can be approximated 
by a simple tilt, where the maximum difference in the phase across the region is:

$$
\Delta_{\Omega}=\max \left(P(x, y)_{x, y \in \Omega} \quad\right)-\min \left(P(x, y)_{x, y \in \Omega} \quad\right)
$$

The number of steps along either horizontal or vertical step is $\mathrm{S}=\mathrm{m} / \mathrm{l}$. Then the number of steps for one wave of tilt (q) in region $\Omega$ is approximately

$$
q_{\Omega}=S / \Delta_{\Omega}=\frac{m}{l \Delta_{\Omega}}
$$

With such approximation, the efficiency term $\eta_{a b e r r}, \Omega$ within region $\Omega$ can be estimated for a 2-D aberration.

$$
\eta_{a b e r r, \Omega}=\left(\frac{\sin \left(\pi / q_{\Omega}\right)}{\pi / q_{\Omega}}\right)^{2}=\left(\frac{\sin \left(\pi l \Delta_{\Omega} / m\right)}{\pi l \Delta_{\Omega} / m}\right)^{2}
$$

If we assume the fractional intensity of the beam on region $\Omega$ is $\mathrm{W}_{\Omega}$, then the total efficiency across the whole aperture is the weighted average of $\eta_{\text {aberr },}$, across the whole aperture as in Equation (7) ${ }^{[12]}$.

$$
\eta_{\text {aberr }}=\sum_{\Omega} W_{\Omega} \eta_{\text {aberr }, \Omega}=\sum_{\Omega} W_{\Omega}\left(\frac{\sin \left(\pi / q_{\Omega}\right)}{\pi / q_{\Omega}}\right)^{2}=\sum_{\Omega} W_{\Omega}\left(\frac{\sin \left(\pi l \Delta_{\Omega} / m\right)}{\pi l \Delta_{\Omega} / m}\right)^{2}
$$

it is also clear that if the resolution of the correction device is high enough, this efficiency $\eta_{\text {aber }}$ can be very high.

The fourth factor $\eta_{S L M}$ is the efficiency associated with the non-ideal nature of the correction device, the LC SLM ${ }^{[13,14,15]}$. Consider a simple case of a tip-tilt corrector, to correct for hundreds of waves of aberration, the thickness of the LC-SLM may become impractically large if the LC-SLM needs to produce such large Optical Path Difference (OPD), because the birefringence of the liquid crystal material is limited. For monochromatic light, this limitation is resolved by considering an approach where the phase profile generated by the LC-SLM is a modulo $2 \pi$ version of the desired phase profile. In this case, the 
maximum OPD change required at any point on the LC-SLM is only $\lambda$. ( $\lambda$ here is the wavelength of light.). The phase profile on a LC-SLM is a stair-like blazed grating with phase reset of $2 \pi$. As has been discussed extensively by ${ }^{[4,14]}$, most pixels in such stair-like blazed grating can produce a phase profile very close to the desired phase profile. However, the orientation of the liquid crystal director cannot undergo abrupt change to produce the phase discontinuity at the phase reset region. There is a fly-back region where the phase slope is in the opposite direction to the desired phase slope. The efficiency of the LC-SLM $\eta_{S L M}$ is related to the ratio of the width of the fly-back region and the grating period as expressed in Equation $8^{[3]}$. A power of two in Equation 4 is to take into account the efficiency for coherent light.

$$
\eta_{S L M} \approx\left(1-\frac{\Lambda_{F}}{\Lambda}\right)^{2}
$$

Here $\Lambda$ is the width of each grating segment, $\Lambda_{F}$ is the width of the fly-back region. In a 2-D LC-SLM discussed in this article, the exact value of this efficiency term depends on many factors such as the cell thickness, liquid crystal material birefringence, aperture ratio of the electrode etc.

However we find $\Lambda_{F}$ is primarily related to the strength of the fringing electric field, which is related to the cell thickness of the device. Assume the width of the fly-back region is related to the cell thickness by an equation of the form shown in Equation 9:

$$
\Lambda_{F}=a\left(\frac{d}{P S}\right)^{\beta}
$$

Here $a$ is a constant related to the strength of the fringing electric field. $d$ is the thickness of the liquid crystal device, PS is the pixel spacing of the LC-SLM. We can fit the diffraction efficiency data obtained by director simulation and FDTD simulation ${ }^{[16]}$ using Equation 10. 


$$
\eta=\left(1-\frac{\Lambda_{F}}{\Lambda}\right)^{2}=\left(1-\frac{a(d / P S)^{\beta}}{\bar{N}}\right)^{2}
$$

Here $\bar{N}$ is the average number of pixels for every wave of aberration. The fitted efficiency agrees excellently with the FDTD simulation data, for cases where voltage optimization is carried out, with the coefficient $a$ as 0.85 , and $\beta$ as 0.7 .

Finnaly we have:

$$
\begin{aligned}
& \eta_{\text {total }}=\eta_{s} \eta_{\text {ray }} \eta_{\text {aber }} \eta_{S L M} \\
&=\eta_{s}\left(1-u_{0}\right)\left[\sum_{\Omega} W_{\Omega}\left(\frac{\sin \left(\pi l \Delta_{\Omega} / m\right)}{\pi l \Delta_{\Omega} / m}\right)^{2}\right]\left(1-\frac{a(d / P S)^{\beta}}{\bar{N}}\right)^{2}
\end{aligned}
$$

With the above discussion, it is possible to relate the total optical throughput of the system to many design parameters as in Equation 11. For example, the total optical throughput is a function of the scattering and reflection loss in the system $\left(\eta_{s}\right)$, the extent of ray missing corresponding pixel $\left(u_{0}\right)$, the resolution of the correction device $(\mathrm{m})$, the complexity of the wavefront $\left(\Delta_{\Omega}\right)$, the pixel spacing of the correction device (PS) and cell thickness of the device (d), etc.

\section{ACKNOWLEDGEMENTS}

This work is supported by NASA space communication project and DARPA TeraHertz Operational Reachback THOR 
project. The author thanks Michael Fisch for advising and helpful discussion.

\section{REFERENCES}

[1] M.T. Gruneisen, L.F. DeSandre, J.R. Rotge, R.C. Dymale, D.L. Lubin, "Programmable diffractive Optics for wide-dynamic-range wavefront control using liquid crystal spatial light modulators”, Opt. Eng. 43 (6), p1378, (2004) .

[2] M.T. Gruneisen, R.C. Dymale, J.R. Rotge, L.F. DeSandre, D.L. Lubin, “Compensated telescope system with programmable diffractive optic,” Opt. Eng. 44 (2), (2005).

[3] M.T. Gruneisen, L.F. DeSandre, D.L. Lubin, R.C. Dymale, J.R. Rotge, "Wavelength-Dependent Charactoristics of a Telescope System with Diffractive Wavefront Compensatioin,” Opt. Eng. 44 (2), (2005).

[4] P. F. Mcmanamon, E.A. Watson, T. A. Dorschner, et al, "Application look at the use of liquid crystal writeable gratings for steering passive radiation,” Opt. Eng., 32,2657 (1993).

[5] G.D. Love, "Wavefront correction of Zernike modes with a Liquid-crystal spatial light modulator", Appl. Opt. 36, 1517 (1997).

[6] D. Dayton, S. Restaino, J.Gonglewski, "Novel Spatial Light Modulators for Active and Adaptive Optics," SPIE Proc., 4124 (2000)

[7] L.J. Friedman, D.S. Hobbs, S. Lieberman, D.L. Corkum, H.W. Nguyen, R.C. Sharp, T.A. Dorschner "Spatially resolved phase imaging of a programmable liquid-crystal grating”, Appl. Opt., 35 (31), p6236 (1996).

[8] V.G. Dominic, E.A.Watson, "Measurement and modeling of the angular dispersion in liquid crystal 
broadband beam steering devices", Opt. Eng. 35 (12), p3371 (1996).

[9] X. Wang, D. Wilson, R. Muller, P. Maker, D. Psaltis, "Liquid-crystal blazed-grating beam deflector", Appl. Opt. 39 (35), p6545 (2000).

[10] D. P. Resler, D.S. Hobbs, R.C. Sharp, L.J. Friedman, and T. A. Dorschner, "High-efficiency liquid-crystal optical phased-array beam steering”, Opt. Letts., 21 (9), p689 (1996).

[11] R.M Matic,"Bazed phase liquid crystal beam steering", SPIE Proc. 2120, p194, (1994)

[12] J.W. Goodman, Introduction to Fourier Optics, p210, ISBN 0-07-024254-2, Second Edition, McGraw-Hill Companies Inc, 1988.

[13] X. Wang, B. Wang, J.J. Pouch, F.A. Mirranda, J. E. Anderson, P.J. Bos,"Liquid Crystal on Silicon (LC-SLM) Wavefront Corrector and Beam Steerer", Proc. SPIE 5162, (2003).

[14] X. Wang, B. Wang, P.J. BOS, P. F. McManamon, J.J. Pouch, F.A. Mirranda, J. E. Anderson, "Modeling and Design of an Optimized Liquid Crystal Optical Phased Array" Submitted to J Appl. Phys. [15] J. E. Anderson, P.E. Watson, and P.J. Bos, Liquid Crystal Display 3-D Director Simulator Software and Technical Guide (Artech House, Norwood, Mass., 2001).

[16] X. Wang, B. Wang, P. J. Bos, J. E. Anderson, J.J. Pouch, F.A. Mirranda, "FDTD simulation of a Liquid Crystal Optical Phased Array,” J. Opt. Soc. Am. A, Vol. 22, (2), (2005). 